Proyecciones

Vol. 19, No 3, pp. 249-269, December 2000.

Universidad Católica del Norte

Antofagasta - Chile

DOI: 10.4067/S0716-09172000000300003

\title{
DIBARIC ALGEBRAS
}

\author{
MARIA APARECIDA COUTO * \\ Universidade Federal do Río Grande do Norte, Brazil \\ and \\ JUAN C. GUTIÉRREZ FERNÁNDEZ ${ }^{\dagger}$ \\ Universidade de São Paulo, Brazil
}

\begin{abstract}
Here we give basic properties of dibaric algebras which are motivated by genetic models. Dibaric algebras are not associative and they have a non trivial homomorphism onto the sex differentiation algebra. We define first join of dibaric algebras next indecomposable dibaric algebras. Finally, we prove the uniqueness of the decomposition of a dibaric algebra, with semiprincipal idempotent, as the join of indecomposable dibaric algebras.
\end{abstract}

KEY WORDS : Nonassociative algebras, baric algebras, genetic algebras, dibaric algebras.

1991 AMS Subject classification : 17D92.

\footnotetext{
*Partially supported by CAPES-PICD process BEX0063/98-3.
}

${ }^{\dagger}$ Partially supported by DGICYT process PB97-1291-C03-01. 


\section{Introduction}

The study of dibaric algebras has as motivation the algebras coming from genetic models in bisexual populations with sex linked genetic inheritance. First, Etherington [3], introduced the idea of treating the male and female components of a population separately and next Holgate [4] formalized this concept with the introduction of the sex differentiation algebra and dibaric algebras. Following the modern notation of Wörz-Busekros [7], we introduce Holgate's definitions bellow. See also the survey [6] for more information. Here $F$ will be a field of characteristic different from two.

Let $\S$ be a bi-dimensional commutative $F$-algebra generated by the elements $\{\mathrm{m}, \mathrm{f}\}$, and with multiplication table $\mathrm{m}^{2}=0, \mathrm{mf}=\mathrm{fm}=$ $(\mathrm{m}+\mathrm{f}) / 2$, and $\mathrm{f}^{2}=0$. This algebra $\S$ is called sex differentiation algebra. Now, an algebra $\mathcal{A}$ will be called dibaric if it admits a homomorphism onto the sex differentiation algebra.

Recall that an $F$-algebra is called baric if it admits a homomorphism onto the field $F$. Since $\S^{2}=\langle\mathrm{m}+\mathrm{f}\rangle_{F}$ is an ideal of $\S$ isomorphic to $F$ we get that $\S^{2}$ is a baric algebra and hence we obtain the following well known result

Lemma 1.1. : If an algebra $\mathcal{A}$ is dibaric, then $\mathcal{A}^{2}$ is baric.

Example 1.1. : Let $(, \omega)$ be a baric $\mathbf{R}$-algebra, that is, $\mathcal{B}$ is an $\mathbf{R}$-algebra and $\omega: \mathcal{B} \longrightarrow \mathbf{R}$ is a homomorphism different from zero. Consider $T: \mathcal{B} \longrightarrow \mathcal{B}$ a linear mapping satisfying $\omega \circ T=\omega$. Thus $T$ leaves the ideal $\operatorname{ker}(\omega)$ invariant. Now, we introduce the vector space $\mathcal{A}:=\mathcal{B} \otimes \mathcal{B} \oplus \mathcal{B}$, where $\oplus$ denotes the direct sum and $\otimes$ denotes the tensor product of vector spaces. We identify the elements $x \otimes y \oplus 0 \in \mathcal{A}$ with $x \otimes y \in \mathcal{B} \otimes \mathcal{B}$ and the elements $0 \oplus z \in \mathcal{A}$ with $z \in \mathcal{B}$. In this space we introduce a commutative multiplication by

$$
\begin{gathered}
\left(x_{1} \otimes y_{1}\right)\left(x_{2} \otimes y_{2}\right)=0, z_{1} z_{2}=0 \\
(x \otimes y) z=\frac{1}{2}(x y \otimes T(z) \oplus \omega(z) x y) .
\end{gathered}
$$

The algebra $\mathcal{A}$ is the sex linked duplicate of the algebra $\mathcal{B}$ with respect to the linear mapping $T$ (see [7] for more information). Obviously, $\mathcal{A}$ 
is a dibaric algebra with weight $\gamma: \mathcal{A} \longrightarrow \mathcal{S}$ defined by $\gamma(x \otimes y \oplus z):=$ $\omega(x y) h+\omega(z) m$.

Example 1.2. : Let $A=A_{h} \oplus A_{m}$ be the 5-dimensional commutative $\mathbf{R}$-algebra with $\mathbf{a}_{1}, \mathbf{a}_{2}$ and $\mathbf{a}_{3}$ as basis of $A_{h}$, with $\mathbf{b}_{1}$ and $\mathbf{b}_{2}$ as basis of $A_{m}$ and with multiplication table as follows: $A_{h}^{2}=0, A_{m}^{2}=0$ and (for $i, j=1,2$ )

$$
\mathbf{a}_{i} \mathbf{b}_{j}=\frac{1}{2}\left(\delta_{i j} \mathbf{a}_{i}+\left(1-\delta_{i j}\right) \mathbf{a}_{3}+\mathbf{b}_{i}\right), \quad \mathbf{a}_{3} \mathbf{b}_{j}=\frac{1}{2}\left(\mathbf{a}_{1} \mathbf{b}_{j}+\mathbf{a}_{2} \mathbf{b}_{j}\right)
$$

where $\delta_{i j}$ is equal to 1 if $i=j$ and is equal to 0 in another case. The algebra $A$ is dibaric with weight $\gamma: A \longrightarrow S$ defined by $\gamma\left(x_{1} \mathbf{a}_{1}+\right.$ $\left.x_{2} \mathbf{a}_{2}+x_{3} \mathbf{a}_{3}+y_{1} \mathbf{b}_{1}+y_{2} \mathbf{b}_{2}\right):=\left(x_{1}+x_{2}+x_{3}\right) h+\left(y_{1}+y_{2}\right) m$. This algebra is called the zygotic algebra for sex linked inheritance for two alleles with simple Mendelian segregation rates. We claim, without proof, the following relevant fact: every element $x \in A$ with $\gamma(x)=$ $h+m$ satisfies the plenary train equation $\left[8 \mathrm{x}^{[5]}-6 x^{[4]}-3 x^{[3]}+\right.$ $\left.x^{[2]}=0\right]$ wheretheplenarypower saredefinedinductivelyby $\mathrm{x}^{[1]}=x$ and $x^{[k+1]}=x^{[k]} x^{[k]}$ for $k \geq 1$. Therefore, if $x \in A$ represents a state of a population $(\gamma(x)=h+m)$, then its trajectory $\left\{x^{[k]}\right\}_{k=1}^{\infty}$ converge and $x^{[\infty]}=\lim _{k l \infty} x^{[k]}$ is equal to the idempotent $\left(8 x^{[4]}+2 x^{[3]}-x^{[2]}\right) / 9$. We notice that an explicit form of $x^{[\infty]}$, in terms of the corresponding gametic algebra, was given by Lyubich in [5] (see also [7, 8, 9] for more information). Finally, we claim that $8 x^{[4]}-6 x^{[3]}-3 x^{[2]}+x^{[1]} \in$ $\operatorname{ann}(A)=\mathbf{R}\left\langle\mathbf{a}_{1}+\mathbf{a}_{2}-2 \mathbf{a}_{3}\right\rangle$ for all $x \in A$ with $\gamma(x)=\mathrm{f}+m$.

\section{Dibaric Weight Homomorphisms}

In the following $\mathcal{A}$ will be an algebra (not necessarily commutative or associative) over the field $F$. A function $\gamma: \mathcal{A} \longrightarrow \S$, where $\S$ is the sex differentiation algebra defined above, is called dibaric weight homomorphism if $\gamma$ is an onto homomorphism of algebras. So, if $a, b$ are elements in $\mathcal{A}$ such that $\gamma(a)=\mathrm{m}$ and $\gamma(b)=\mathrm{f}$, then we have the following decomposition

$$
\mathcal{A}=F a \oplus F b \oplus \operatorname{ker}(\gamma)
$$


where $\operatorname{ker}(\gamma):=\{x \in \mathcal{A}: \gamma(x)=0\}$ is an ideal of $\mathcal{A}$ of codimension two.

Notice that for every dibaric weight homomorphism $\gamma$ and every automorphism $f: \S \longrightarrow \S$, the mapping $f \circ \gamma$ is a dibaric weight homomorphism and $\operatorname{ker}(\gamma)=\operatorname{ker}(f \circ \gamma)$. We say that two dibaric weight homomorphisms $\gamma$ and $\gamma^{\prime}$ are equivalent if there exists an automorphism $f: \S \longrightarrow \S$ such that $\gamma^{\prime}=f \circ \gamma$.

Lemma 2.1. : The sex differentiation algebra has only two automorphisms, the identity and the involution $*: \S \longrightarrow \S$ given by $*(m)=\mathrm{f}, *(\mathrm{f})=m$.

Proof. Let $f: \S l \S$ be an onto homomorphism. Then $0=f\left(\mathrm{~m}^{2}\right)=$ $f(\mathrm{~m})^{2}$, and analogously, $0=f(\mathrm{f})^{2}$ and hence either $f(\mathrm{~m}) \in F \mathrm{~m}$, $f(\mathbf{f}) \in F \mathrm{f}$ or $f(\mathrm{~m}) \in F \mathrm{f}, f(\mathbf{f}) \in F \mathrm{~m}$. Next using that $f(\mathrm{~m}) f(\mathbf{f})=$ $f(m h)=f((\mathrm{~m}+\mathbf{f}) / 2)=(f(\mathrm{~m})+f(\mathbf{f})) / 2$ we get the result.

From this result, it follows that each equivalence class defined above has exactly two weight. So, if $\gamma$ and $\gamma^{\prime}$ are two different and equivalent dibaric weight homomorphisms, then $\gamma^{\prime}=* \circ \gamma$. We denote by $\&$ the set of these equivalence classes, that is, an element of $\mathscr{E}$ is $\left\{\gamma, \gamma^{*}\right\}$, where $\gamma$ is a dibaric weight homomorphism and $\gamma^{*}:=* \circ \gamma$.

Theorem 2.1. : The application $\left\{\gamma, \gamma^{*}\right\} \longmapsto \operatorname{ker}(\gamma)$ is a bijection between the set $\mathscr{E}$ of equivalence class of dibaric weight homomorphisms of $\mathcal{A}$, and the set of ideals $I$ of $\mathcal{A}$ of codimension two, such that $\mathcal{A} / I \cong \S$.

Corollary 2.1. : Dibaric weight homomorphisms with same kernel are equivalent.

Lemma 2.2. : Different dibaric weight homomorphisms of an algebra $\mathcal{A}$ are linearly independent. 
Proof. Let $\gamma_{1}, \gamma_{2}, \cdots, \gamma_{m}$ be different dibaric weight homomorphisms of $\mathcal{A}$ and consider scalars $\alpha_{1}, \cdots, \alpha_{m}$ in $F$ such that

$$
\alpha_{1} \gamma_{1}(z)+\alpha_{2} \gamma_{2}(z)+\cdots+\alpha_{m} \gamma_{m}(z)=0
$$

for all $z \in \mathcal{A}$. We will prove that $\alpha_{1}=0=\alpha_{2}=\cdots=\alpha_{m}$ using induction over the number $m$ of different dibaric weight homomorphisms of $\mathcal{A}$. The case $m=1$ is trivial. Let $m>1$. Then by hypothesis of induction, the lemma is true for $m-1$ weights.

Notice that if there exists and index $i$ such that $\alpha_{i}=0$, then by hypothesis of induction, we obtain that $\alpha_{j}=0$ for $j=1,2, \ldots, m$, and the result follows.

First, we suppose that all weight homomorphisms have same kernel. Under this assumption, we obtain from Corollary 2.1 that $m=2$ and $\gamma_{2}=\gamma_{1}^{*}$. Now, let $z \in \mathcal{A}$ such that $\gamma_{1}(z)=\mathrm{m}$. Then $0=$ $\alpha_{1} \gamma_{1}(z)+\alpha_{2} \gamma_{1}^{*}(z)=\alpha_{1} \mathrm{~m}+\alpha_{2} \mathrm{f}$, and hence it follows that $\alpha_{1}=0=\alpha_{2}$.

Finally, we suppose that there exist homomorphisms with different kernels. We can assume that $\operatorname{ker}\left(\gamma_{1}\right) \neq \operatorname{ker}\left(\gamma_{2}\right)$. Under this condition, consider $x \in \mathcal{A}$ such that $\gamma_{1}(x) \neq 0$ and $\gamma_{2}(x)=0$. Since im $\left(\gamma_{1}\right)=\mathcal{S}$, there exists $y \in \mathcal{A}$ such that $\mathrm{m}+\mathrm{f}=\gamma_{1}(x) \gamma_{1}(y)=\gamma_{1}(x y)$. Multiplying the equation $(2.2)$ by $\gamma_{1}(x y)$, we obtain

$$
\alpha_{1} \gamma_{1}(x y) \gamma_{1}(z)+\alpha_{2} \gamma_{1}(x y) \gamma_{2}(z)+\cdots+\alpha_{m} \gamma_{1}(x y) \gamma_{m}(z)=0
$$

and replacing $z \rightarrow(x y) z$ in equation $(2.2)$ we get

$$
\alpha_{1} \gamma_{1}(x y) \gamma_{1}(z)+\alpha_{2} \gamma_{2}(x y) \gamma_{2}(z)+\cdots+\alpha_{m} \gamma_{m}(x y) \gamma_{m}(z)=0
$$

for all $z \in \mathcal{A}$. Next subtracting the equation (2.4) from equation (2.3), we get

$$
\alpha_{2}\left(\gamma_{1}(x y) \gamma_{2}(z)+\cdots+\alpha_{m}\left(\gamma_{1}(x y)-\gamma_{m}(x y)\right) \gamma_{m}(z)=0\right.
$$

Notice that $\gamma_{2}(x y)=0$.Since $\gamma_{k}(x y) \in \gamma_{k}\left(\mathcal{A}^{2}\right)=\S^{2}=\langle\mathrm{m}+\mathrm{f}\rangle_{F}$ for $k=1, \ldots, m$, there exist scalars $\beta_{k}$ such that $\gamma_{k}(x y)=\beta_{k}(\mathrm{~m}+\mathrm{f})$. So, the equation (2.5) can be written as follows

$$
(\mathrm{m}+\mathrm{f})\left(\alpha_{2} \gamma_{2}(z)+\alpha_{3}\left(1-\beta_{3}\right) \gamma_{3}(z)+\cdots+\alpha_{m}\left(1-\beta_{m}\right) \gamma_{m}(z)\right)=0
$$


and therefore

$\gamma(z):=\alpha_{2} \gamma_{2}(z)+\alpha_{3}\left(1-\beta_{3}\right) \gamma_{3}(z)+\cdots+\alpha_{m}\left(1-\beta_{m}\right) \gamma_{m}(z) \in F(\mathrm{~m}-\mathrm{f})$.

Thus, $\gamma\left(\mathcal{A}^{2}\right) \in F(\mathrm{~m}+\mathrm{f}) \cap F(\mathrm{~m}-\mathrm{f})=(0)$. Now, if $\gamma(z)=\lambda_{z}(\mathrm{~m}-\mathrm{f})$ then $0=\gamma\left(z^{2}\right)=\gamma(z)^{2}=-\lambda_{z}^{2}(\mathrm{~m}+\mathrm{f})$. This implies that $\lambda_{z}=0$ and hence $\gamma(z)=0$. Using the hypothesis of induction on $\gamma(z)=0$, we have $\alpha_{2}=0$. So, $\alpha_{j}=0$ for all $j$.

From the above result, it follows that the number of different dibaric weight homomorphisms of an algebra $\mathcal{A}$ is at most $n$, where $n$ is the dimension of $\mathcal{A}$. We will show that this bound can be improved. For an algebra $\mathcal{A}$ we define inductively

$$
\mathcal{A}^{[1]}=\mathcal{A}, \quad \mathcal{A}^{[i]}=\mathcal{A}^{[i-1]} \mathcal{A}^{[i-1]}, \quad i>1 .
$$

So, if $\mathcal{A}$ has finite dimension, there exists a natural number $r$, such that $\mathcal{A}^{[r+1]}=\mathcal{A}^{[r]}$. Under this condition, we can show that the number of different dibaric weight homomorphisms of $\mathcal{A}$ is at most $2 \cdot \operatorname{dim}\left(\mathcal{A}^{[r]}\right)$. Notice that for a dibaric algebra $\mathcal{A}^{2} \neq \mathcal{A}$.

According to Lemma 1.1, if $\mathcal{A}$ is a dibaric algebra with $\gamma$ as dibaric weight homomorphism, then $\mathcal{A}^{2}$ is baric and $\hat{\gamma}: \mathcal{A}^{2} \longrightarrow \S^{2}$, the restriction of $\gamma: \mathcal{A} \longrightarrow \S$ is a baric weight homomorphism for $\mathcal{A}^{2}$. From, now on we identify $\S^{2}$ with the field $F$.

Theorem 2.2. : The application $\left\{\gamma, \gamma^{*}\right\} \longmapsto \hat{\gamma}$ is an injection between the set $\mathscr{E}$ of equivalence classes of dibaric weight homomorphism of $\mathcal{A}$ and the set of baric weight homomorphisms of $\mathcal{A}^{2}$.

Proof. First, we note that the elements of $\S^{2}$ are invariant by the involution $*$ and hence $p q=*(p q)=*(p) *(q)$ for all $p, q \in \S$. From this fact, we obtain that the application is well defined, that is $\hat{\gamma}=\hat{\gamma}^{*}$.

Next, we will show that the application is injective. Let $\tau, \gamma$ be two dibaric weight homomorphisms, such that $\hat{\tau}=\hat{\gamma}$. We have to show that these two homomorphisms are equivalent but according to Corollary 2.1, it suffices to show that they have the same kernels. So, let $a \in \operatorname{ker}(\gamma)$. Since $\operatorname{ker}(\gamma)$ is an ideal of $\mathcal{A}$, we have that $a \mathcal{A} \subseteq$ $\operatorname{ker}(\gamma) \cap \mathcal{A}^{2}$ and using the hypothesis, we have $a \mathcal{A} \subseteq \operatorname{ker}(\tau)$. Then, it 
follows that $a \in \operatorname{ker}(\tau)$, since in other case we have an element $b \in \mathcal{A}$, such that $\tau(a b) \neq 0$ and this is a contradiction. So, we showed that $\operatorname{ker}(\gamma) \subset \operatorname{ker}(\tau)$ and therefore $\operatorname{ker}(\gamma)=\operatorname{ker}(\tau)$. This implies that the two homomorphisms are equivalent.

In an analogous way we can prove the following lemma

Lemma 2.3. : The application $\omega \longmapsto \hat{\omega}$ is an injection between the set of baric weight homomorphisms of a baric algebra $\mathcal{B}$ and the set of baric homomorphisms of $\mathcal{B}^{2}$.

Proof. Let $\omega, \tau: B \longrightarrow F$ be two baric weight homomorphisms of $\mathcal{B}$ such that $\omega(x)=\tau(x)$ for all $x \in \mathcal{B}^{2}$. We already know that $\omega=\tau$ if and only if $\operatorname{ker}(\omega)=\operatorname{ker}(\tau)$. If $x \in \operatorname{ker}(\omega)$, then $x^{2} \in \operatorname{ker}(\omega) \cap \mathcal{A}^{2}=$ $\operatorname{ker}(\tau) \cap \mathcal{A}^{2}$ and hence $0=\tau\left(x^{2}\right)=\tau(x)^{2}$. This forces $\tau(x)=0$. Thus, we have proved that $\operatorname{ker}(\omega) \subset \operatorname{ker}(\tau)$ that is $\operatorname{ker}(\omega)=\operatorname{ker}(\tau)$ and hence by Lemma 3.3.1 of [5] we have that $\omega=\tau$.

According to [5] the number of baric weight homomorphisms of a baric algebra $\mathcal{B}$ is at most its dimension. Using this fact, Lemma 2.3 and Theorem 2.2 we have the following result:

Corollary 2.2. : Let $\mathcal{A}$ be a dibaric algebra of dimension $n$ and $r$ a natural number such that $\mathcal{A}^{[r+1]}=\mathcal{A}^{[r]}$. Under these conditions, the number of different dibaric weight homomorphisms of $\mathcal{A}$ is at must $2 \cdot \operatorname{dim}\left(\mathcal{A}^{[r]}\right)$.

Lemma 2.4. : Let $\mathcal{A}$ be a dibaric algebra with $\gamma$ as dibaric weight homomorphism. If there exists a monomial $p(x) \in F[x], p(x) \neq$ 0 , such that $p(a)=0$, for all $a \in \operatorname{ker}(\gamma)$, then the only dibaric weight homomorphisms of $\mathcal{A}$ are $\gamma$ and $\gamma^{*}$.

Proof. Let $\tau: \mathcal{A} \longrightarrow \S$ be a dibaric weight homomorphism. If $\tau(a) \neq 0$, then there exists $b \in \mathcal{A}$ such that $\mathrm{m}+\mathrm{f}=\tau(a) \tau(b)=$ $\tau(a b)$. Then $\tau(p(a b))=p(\tau(a b))=p(\mathrm{~m}+\mathrm{f})=\mathrm{m}+\mathrm{f}$ and hence $a b \notin$ $\operatorname{ker}(\gamma)$. This forces that $a \notin \operatorname{ker}(\gamma)$. Consequently, $\operatorname{ker}(\gamma) \subset \operatorname{ker}(\tau)$ and $\operatorname{ker}(\gamma)=\operatorname{ker}(\tau)$. Now the result follows form Theorem 2.1. 
Example 2.1. : An important example for biological applications is the evolution algebra $\mathcal{A}_{V}$ described in [5, Cap.I]. Consider two positive integer $n$ and $\nu$ and real scalars $p_{i j, k}^{(m)}$ and $p_{i j, l}^{(h)}$ satisfying

$$
p_{i j, k}^{(m)} \geq 0, \quad p_{i j, l}^{(h)} \geq 0, \quad \sum_{k=1}^{n} p_{i j, k}^{(m)}=1, \quad \sum_{l=1}^{\nu} p_{i j, l}^{(h)}=1
$$

for $1 \leq i \leq n, 1 \leq j \leq \nu$. Now we define in the space $\mathbf{R}^{n} \times \mathbf{R}^{\nu}$ a commutative product as follows

$$
e_{i} e_{k}=0, \quad e_{i} \bar{e}_{j}=\frac{1}{2}\left(\sum_{k=1}^{n} p_{i j, k}^{(m)} e_{k}+\sum_{l=1}^{\nu} p_{i j, l}^{(f)} \bar{e}_{l}\right), \quad \bar{e}_{j} \bar{e}_{l}=0
$$

where we identify $e_{i} \equiv\left(e_{i}, 0\right), \bar{e}_{j} \equiv\left(0, \bar{e}_{j}\right)$ such that $\left(e_{i}\right)_{i=1}^{n}$ is a canonical basis of $\mathbf{R}^{n}$ and $\left(\bar{e}_{j}\right)_{j=1}^{\nu}$ is a canonical basis of $\mathbf{R}^{\nu}$. In this way, we obtain a commutative algebra $\mathcal{A}_{V}$. The following result is well known

Lemma 2.5. : The mapping $s: \mathcal{A}_{V} \longrightarrow \oint$ given by $s(z)=$ $\left(\sum_{i=1}^{n} x_{i}\right) m+\left(\sum_{j=1}^{\nu} y_{j}\right) \mathrm{f}$ where $z=(x, y) \in \mathcal{A}_{V}$ is a dibaric weight homomorphism.

Lemma 2.6. : The weight homomorphism $s: \mathcal{A}_{V} \longrightarrow \oint$ is characterized, up to equivalence, as the only positive dibaric weight homomorphism in the sense that the image of $\Omega=\left\{(x, y) \in \mathcal{A}_{V}\right.$ : $\left.x_{i}, y_{j} \geq 0, \sum_{i} x_{i}=1, \sum_{j} y_{j}=1\right\}$ is contained in the set $\{\alpha m+\beta \mathrm{f}$ $\alpha, \beta \geq 0, \alpha+\beta>0\}$.

Proof. Let $\gamma: \mathcal{A}_{V} \longrightarrow \S$, be a positive dibaric weight homomorphism. For $1 \leq i \leq n$ and $1 \leq j \leq \nu$ we have that $\gamma\left(e_{i}\right), \gamma\left(\bar{e}_{j}\right) \in \S$, so

$$
\gamma\left(e_{i}\right)=\alpha_{i} \mathrm{~m}+\beta_{i} \mathrm{f}, \quad \gamma\left(\bar{e}_{j}\right)=\bar{\alpha}_{j} \mathrm{~m}+\bar{\beta}_{j} \mathrm{f},
$$

where $\alpha_{i}, \beta_{i}, \bar{\alpha}_{j}, \bar{\beta}_{j} \in \mathbf{R}$. Then, because $\left(e_{i}\right)^{2}=0$, we get $0=\gamma\left(e_{i}^{2}\right)=$ $\gamma\left(e_{i}\right)^{2}=\left(\alpha_{i} \mathrm{~m}+\beta_{i} \mathrm{f}\right)^{2}=\alpha_{i} \beta_{i}(\mathrm{~m}+\mathrm{f})$ and analogously, using that $\left(\bar{e}_{j}\right)^{2}=0$, we obtain that $0=\bar{\alpha}_{j} \bar{\beta}_{j}(\mathrm{~m}+\mathrm{f})$. On the other hand, the elements $2 e_{i} \bar{e}_{j}$ and $e_{i}+\bar{e}_{j}$ belong to $\Omega$ and their images are 
$\gamma\left(2 e_{i} \bar{e}_{j}\right)=2 \gamma\left(e_{i}\right) \gamma\left(\bar{e}_{j}\right)=2\left(\alpha_{i} \mathrm{~m}+\beta_{j} \mathrm{f}\right)\left(\bar{\alpha}_{j} \mathrm{~m}+\bar{\beta}_{j} \mathrm{f}\right)=\left(\alpha_{i} \bar{\beta}_{j}+\beta_{i} \bar{\alpha}_{j}\right)(\mathrm{m}+\mathrm{f})$ and $\gamma\left(e_{i}+\bar{e}_{j}\right)=\left(\alpha_{i}+\bar{\alpha}_{j}\right) \mathrm{m}+\left(\beta_{i}+\bar{\beta}_{j}\right) \mathrm{f}$. Therefore, we have the following relations,

$\alpha_{i} \beta_{i}=0, \quad \alpha_{i}+\bar{\alpha}_{j}>0, \quad \alpha_{i} \bar{\beta}_{j}+\beta_{i} \bar{\alpha}_{j}>0, \quad \beta_{i}+\bar{\beta}_{j}>0, \quad \bar{\alpha}_{j} \bar{\beta}_{j}=0$.

In particular $\alpha_{1} \beta_{1}=0$ and hence either $\alpha_{1} \neq 0$ and $\beta_{1}=0$ or $\alpha_{1}=0$ and $\beta_{1} \neq 0$. We will consider the two cases separately. In the first case, we will prove that $\gamma=s$ and in the second case that $\gamma=* \circ s$.

First, we suppose that $\alpha_{1} \neq 0$ and $\beta_{1}=0$. Then, for each $j$, the equation $\alpha_{1} \bar{\beta}_{j}+\beta_{1} \bar{\alpha}_{j}>0$ implies that $\bar{\beta}_{j} \neq 0$. Therefore, $\bar{\alpha}_{j}=0$. Now, because $\alpha_{1}+\bar{\alpha}_{j}, \beta_{1}+\bar{\beta}_{j}>0$ we obtain that $\alpha_{1}>0$ and $\bar{\beta}_{j}>0$. In particular, $\bar{\beta}_{1}>0$ and $\bar{\alpha}_{1}=0$. So, from inequality $\alpha_{i} \bar{\beta}_{1}+\beta_{i} \bar{\alpha}_{1}>0$ we have that $\alpha_{i}>0$ and hence $\beta_{i}=0$. Thus, we have proved that $\gamma\left(e_{i}\right)=\alpha_{i} \mathrm{~m}$ and $\gamma\left(\bar{e}_{j}\right)=\bar{\beta}_{j} \mathrm{f}$. Then,

$$
\begin{gathered}
\alpha_{i} \bar{\beta}_{j}(m+\mathrm{f})=2 \gamma\left(e_{i}\right) \gamma\left(\bar{e}_{j}\right)=2 \gamma\left(e_{i} \bar{e}_{j}\right)=\gamma\left(\sum_{k=1}^{n} p_{i j, k}^{(m)} e_{k}+\sum_{l=1}^{\nu} p_{i j, l}^{(f)} \bar{e}_{l}\right) \\
=\sum_{k=1}^{n} p_{i j, k}^{(m)} \alpha_{k} m+\sum_{l=1}^{\nu} p_{i j, l}^{(f)} \bar{\beta}_{l} \mathrm{f}
\end{gathered}
$$

So, we obtain the following equalities,

$$
\alpha_{i} \bar{\beta}_{j}=\sum_{k=1}^{n} p_{i j, k}^{(m)} \alpha_{k}, \quad \alpha_{i} \bar{\beta}_{j}=\sum_{l=1}^{n} p_{i j, l}^{(f)} \bar{\beta}_{l},
$$

for $1 \leq i \leq n$ and $1 \leq j \leq \nu$. Now considering the scalars

$$
\begin{array}{ll}
\alpha_{\max }=\max \left(\alpha_{i}\right)_{i=1}^{n}, & \alpha_{\min }=\min \left(\alpha_{i}\right)_{i=1}^{n}, \\
\bar{\beta}_{\max }=\max \left(\bar{\beta}_{j}\right)_{j=1}^{\nu}, & \bar{\beta}_{\min }=\min \left(\bar{\beta}_{j}\right)_{j=1}^{\nu},
\end{array}
$$

and using (2.7), we obtain

$$
\alpha_{\min }=\sum_{k=1}^{n} p_{i j, k}^{(m)} \alpha_{\min } \leq \sum_{k=1}^{n} p_{i j, k}^{(m)} \alpha_{k}=\alpha_{i} \bar{\beta}_{j} \leq \sum_{k=1}^{n} p_{i j, k}^{(m)} \alpha_{\max }=\alpha_{\max } .
$$

and also

$$
\bar{\beta}_{\min }=\sum_{k=1}^{n} p_{i j, k}^{(f)} \bar{\beta}_{\min } \leq \sum_{l=1}^{n} p_{i j, l}^{(f)} \bar{\beta}_{l}=\alpha_{i} \bar{\beta}_{j} \leq \sum_{l=1}^{n} p_{i j, l}^{(f)} \bar{\beta}_{\max }=\bar{\beta}_{\max } .
$$


In particular, $\alpha_{\min } \leq \alpha_{\min } \bar{\beta}_{j}$ and $\alpha_{\max } \bar{\beta}_{j} \leq \alpha_{\max }$, and since all scalars are positive, it follows that $1 \leq \bar{\beta}_{j} \leq 1$, for all $j$. This implies that $\bar{\beta}_{j}=1$. Analogously $\bar{\beta}_{\min } \leq \alpha_{i} \bar{\beta}_{\min }$ and $\alpha_{i} \bar{\beta}_{\max } \leq \bar{\beta}_{\max }$ and then $\alpha_{i}=1$, for all $i$. So, $\gamma\left(e_{i}\right)=\mathrm{m}$ and $\gamma\left(\bar{e}_{j}\right)=\mathrm{f}$. Therefore $\gamma=s$.

Finally, we consider the second case, that is, $\beta_{1} \neq 0$ and $\alpha_{1}=0$. Analogously, we have that $\gamma\left(e_{i}\right)=\beta_{i} \mathrm{f}$ and $\gamma\left(\bar{e}_{j}\right)=\bar{\alpha}_{j} \mathrm{~m}$. Repeating the calculations above with the scalars $\alpha_{i}$ and $\bar{\beta}_{j}$ we get that these are all equal to 1 . Thus, $\gamma=* \circ s$.

So, $s$ and $s^{*}=* \circ s$ are the only positive dibaric weight homomorphisms in this algebra.

\section{Dibaric Algebras}

An ordered pair $(A, \gamma)$, where $A$ is an algebra and $\gamma: A \longrightarrow \S$ is a dibaric weight homomorphism is called dibaric algebra. Under these conditions, the homomorphism is called weight function and the affine subspace $H:=\{x \in \mathcal{A} \mid \gamma(x)=\mathrm{m}+\mathrm{f}\}$ of codimension 2 , is called unit subspace. For each $x \in \mathcal{A}$ with $x^{2} \notin \operatorname{ker}(\gamma)$, we have that $x^{2} / \gamma\left(x^{2}\right) \in$ $H$. We denote the kernel of $\gamma$, by $\mathrm{N}$, that is,

$$
\mathrm{N}=\{x \in \mathcal{A} \mid \gamma(x)=0\}
$$

If $\mathrm{B}$ is any set contained in $\mathcal{A}$, we will denote by $\mathrm{N}_{B}$ the set $\mathrm{N} \cap \mathrm{B}$, that is,

$$
\mathrm{N}_{B}=\{x \in \mathrm{B} \mid \gamma(x)=0\} .
$$

Let $(\mathcal{A}, \gamma)$ be a dibaric algebra. We say that a subalgebra $\mathcal{A}_{1}$ of $\mathcal{A}$ is a dibaric subalgebra of $\mathcal{A}$ if $\mathcal{A}_{1} \cap \operatorname{ker}(\gamma)$ is an ideal of $\mathcal{A}_{1}$ of codimension 2, or equivalently, $\gamma_{1} \equiv \gamma_{\mid \mathcal{A}_{1}}$ is a dibaric weight homomorphism for $\mathcal{A}_{1}$. This subalgebra is denoted by $\left(\mathcal{A}_{1}, \gamma_{1}\right) \subset(\mathcal{A}, \gamma)$. A dibaric algebra $\mathcal{A}$ is not trivial if $\mathrm{N}$ is different from zero, that is, $\mathcal{A}$ is not isomorphic to $\S$.

Also, a subalgebra $\mathcal{A}_{1}$ of $\mathcal{A}$ is called baric subalgebra if $\gamma\left(\mathcal{A}_{1}\right)=$ $\langle\mathrm{m}+\mathrm{f}\rangle_{F}$.

An ideal $I$ is called dibaric ideal if $I \subseteq \operatorname{ker}(\gamma)$, that is, $\gamma_{\mid I}=\{0\}$. Naturally a dibaric ideal cannot be a dibaric subalgebra. We say that a dibaric ideal $I$ is maximal if $I \neq \mathrm{N}$ and the only dibaric ideals of $\mathcal{A}$ 
that contain $I$ are $I$ and $\mathrm{N}$. Notice that the biggest dibaric ideal of a dibaric algebra $(\mathcal{A}, \gamma)$ is $\mathrm{N}$.

The annulator, ann $\mathcal{A}:=\{x \in A \mid x A=(0)\}$ is a dibaric ideal of $\mathcal{A}$. Also, any subspace of ann $\mathcal{A}$ is a dibaric ideal. The ideal $\mathcal{A}^{2}$ of $\mathcal{A}$, is not dibaric, but, according to Lemma 1.1 , we have that $\mathcal{A}^{2}$ is a baric subalgebra.

For any dibaric ideal $I$, we have that the quotient $\mathcal{A} / I$ is a dibaric algebra. It is called dibaric quotient and is denoted by $(\mathcal{A}, \gamma) / I$. The quotient algebra $(\mathcal{A}, \gamma) / \mathcal{N}$ is isomorphic to the sex differentiation algebra $\S$.

Given two dibaric algebras $\left(\mathcal{A}_{1}, \gamma_{1}\right)$ and $\left(\mathcal{A}_{2}, \gamma_{2}\right)$, a dibaric homomorphism of dibaric algebras $f:\left(\mathcal{A}_{1}, \gamma_{1}\right) l\left(\mathcal{A}_{2}, \gamma_{2}\right)$ is a homomorphism of algebras $f: \mathcal{A}_{1} \longrightarrow \mathcal{A}_{2}$ such that $\gamma_{2} \circ f=\gamma_{1}$. For example, the embedding of a dibaric subalgebra and the quotient application are dibaric homomorphisms. Clearly, the composition of dibaric homomorphisms is dibaric. The inverse of a dibaric isomorphism is a dibaric isomorphism, because if $\gamma_{2} \circ f=\gamma_{1}$, this imply that $\gamma_{2}=\gamma_{1} \circ f^{-1}$. We write $\left(\mathcal{A}_{1}, \gamma_{1}\right) \cong\left(\mathcal{A}_{2}, \gamma_{2}\right)$ for isomorphic dibaric algebras, that is, there is a dibaric isomorphism $f:\left(\mathcal{A}_{1}, \gamma_{1}\right) \longrightarrow\left(\mathcal{A}_{2}, \gamma_{2}\right)$.

Every dibaric algebra $(\mathcal{A}, \gamma)$ is not associative because $(\mathcal{A}, \gamma) / \operatorname{ker}(\gamma)$ $\cong \S$ and $\S$ is not associative. In particular, the subalgebra of $\operatorname{End}(\mathcal{A})$ spanned by the left and right multiplication by elements of $\mathcal{A}$, that is $L_{a}(x)=a x$ and $R_{a}(x)=x a$ for all $a, x \in \mathcal{A}$ is not dibaric because it is associative. This associative algebra is called the multiplication algebra of $\mathcal{A}$ and is denoted by $\mathcal{M}(A)$.

Lemma 3.1. : Let $f:\left(\mathcal{A}_{1}, \gamma_{1}\right) \longrightarrow\left(\mathcal{A}_{2}, \gamma_{2}\right)$ be a dibaric homomorphism. Then $\Im(f)$ is a dibaric subalgebra of $\mathcal{A}_{2}$ and $\operatorname{ker}(f)$ is a dibaric ideal of $\mathcal{A}_{1}$. The bijection induced by $f$ is a dibaric isomorphism, that is $\left(\mathcal{A}_{1}, \gamma_{1}\right) / \operatorname{ker}(f) \cong i m(f)$.

Lemma 3.2. : A dibaric homomorphism $f:\left(\mathcal{A}_{1}, \gamma_{1}\right) \longrightarrow\left(\mathcal{A}_{2}, \gamma_{2}\right)$ is an isomorphism if and only if $\hat{f} \equiv f_{\mid \mathcal{N}_{1}}: \mathcal{N}_{1} \longrightarrow \mathcal{N}_{2}$ is an isomorphism, where $\mathcal{N}_{i}=\operatorname{ker}\left(\gamma_{i}\right)$, for $i=1,2$. 
Proof. Let $f:\left(\mathcal{A}_{1}, \gamma_{1}\right) \longrightarrow\left(\mathcal{A}_{2}, \gamma_{2}\right)$ be a dibaric homomorphism such that $\hat{f}: \mathcal{N}_{1} l \mathcal{N}_{2}$ is an isomorphism. Consider $u, v \in \mathcal{A}_{1}$ satisfying $\gamma_{1}(u)=\mathrm{m}$ and $\gamma_{1}(v)=\mathrm{f}$. If $a, b \in \mathcal{A}_{1}$ then there exist elements $x, y \in \mathcal{N}_{1}$ and scalars $\alpha_{1}, \alpha_{2}, \beta_{1}, \beta_{2} \in F$ uniquely determined such that $a=\alpha_{1} u+\alpha_{2} v+x$ and $b=\beta_{1} u+\beta_{2} v+y$. Now we assume that $f(a)=f(b)$. This give us that $f(a-b)=0$, and since $f$ is a dibaric homomorphism, we have that $a-b \in \mathcal{N}_{1}$. This implies that $\alpha_{1}=\beta_{1}$ and $\alpha_{2}=\beta_{2}$. Then, because $f(a)=f(b)$, we obtain that $f(x)=f(y)$. Now by hypothesis $\hat{f}$ is an isomorphism and hence $x=y$. Consequently, $a=b$. The reverse is trivial.

An idempotent element $e$ in a dibaric algebra $(\mathcal{A}, \gamma)$, is called semiprincipal if $e=u+v$, where $\gamma(u)=\mathrm{m}, \gamma(v)=\mathrm{f}$, and $u^{2}=0$, $v^{2}=0$ and $u v=v u=(u+v) / 2$.

Let $(\mathcal{A}, \gamma)$ be a dibaric algebra and $e=u+v$ a semiprincipal idempotent element in $\mathcal{A}$. Then, we have the decomposition $\mathcal{A}=$ $F u \oplus F v \oplus \mathcal{N}$ where $F u \oplus F v$ is a dibaric subalgebra isomorphic to $\S$.

There exists a natural form to get algebras with semiprincipal idempotent elements. If $N$ is an arbitrary algebra over $F$ and $\lambda_{1}, \lambda_{2}, \rho_{1}, \rho_{2}: N \longrightarrow N$ are linear applications, we consider $\mathcal{A}=\S \oplus N$, with the multiplication $\left(\alpha \mathrm{m}+\beta \mathrm{f}, x_{1}\right)\left(\mu \mathrm{m}+\eta \mathbf{f}, x_{2}\right)$ defined by

$$
\left(\frac{(\alpha \eta+\beta \mu)}{2}(\mathrm{~m}+\mathrm{f}), x_{1} x_{2}+\alpha \lambda_{1}\left(x_{2}\right)+\beta \rho_{1}\left(x_{2}\right)+\mu \lambda_{2}\left(x_{1}\right)+\eta \rho_{2}\left(x_{1}\right)\right)
$$

and weight function by $\gamma(\alpha \mathrm{m}+\beta \mathrm{f}, x):=\alpha \mathrm{m}+\beta \mathrm{f}$, where $\alpha, \beta, \mu, \eta \in F$ and $x_{1}, x_{2}, x \in N$. We have that $\gamma$ is different from zero and the element $(\mathrm{m}+\mathrm{f}, 0)$ is a semiprincipal idempotent element of $\mathcal{A}$. This algebra is denoted by $\left[\lambda_{1}, \lambda_{2}, \rho_{1}, \rho_{2}, N\right]$.

Conversely, a dibaric algebra $(\mathcal{A}, \gamma)$ with semiprincipal idempotent $e=u+v$, is isomorphic to $\left[\lambda_{1}, \lambda_{2}, \rho_{1}, \rho_{2}, \mathcal{N}\right]$, where $\mathcal{N}=\operatorname{ker}(\gamma)$,

$$
\lambda_{1}=L_{u \mid \mathcal{N}}, \quad \lambda_{2}=L_{v \mid \mathcal{N}}, \quad \rho_{1}=R_{u \mid \mathcal{N}}, \quad \rho_{2}=R_{v \mid \mathcal{N}} .
$$

The applications $L_{a \mid \mathcal{N}}, R_{a \mid \mathcal{N}}$ denote the restriction of the left and right multiplications by the element $a$ in $\mathrm{N}$, that is, $L_{a}(x)=a x, R_{a}(x)=$ $x a$, for every $x \in \mathrm{N}$.

If $(A, \gamma)$ is a dibaric algebra with semiprincipal idempotent element $e=u+v$, and $I$ is a dibaric ideal of $\mathcal{A}$, then $F u \oplus F v \oplus I$ is a 
dibaric subalgebra of $\mathcal{A}$. Naturally if $I$ is maximal, it follows that this subalgebra is maximal. Conversely, if $I$ is a dibaric ideal, it follows that the subalgebra defined above is maximal.

\section{The Main Theorem}

For two dibaric algebras, $\left(\mathcal{A}_{1}, \gamma_{1}\right)$ and $\left(\mathcal{A}_{2}, \gamma_{2}\right)$, we have the external product $\mathcal{A}_{1} \times \mathcal{A}_{2}$ with the multiplication given by $\left(x_{1}, x_{2}\right)\left(y_{1}, y_{2}\right)=$ $\left(x_{1} y_{1}, x_{2} y_{2}\right)$. This algebra is not necessarily dibaric, but the subspace

$$
\mathcal{A}_{1} \vee \mathcal{A}_{2}:=\left\{\left(x_{1}, x_{2}\right) \in \mathcal{A}_{1} \times \mathcal{A}_{2} \mid \gamma_{1}\left(x_{1}\right)=\gamma_{2}\left(x_{2}\right)\right\}
$$

is a dibaric algebra with dibaric weight homomorphism given by

$$
\gamma_{1} \vee \gamma_{2}\left(x_{1}, x_{2}\right):=\gamma_{1}\left(x_{1}\right)=\gamma_{2}\left(x_{2}\right)
$$

We will call this algebra $\left(\mathcal{A}_{1} \vee \mathcal{A}_{2}, \gamma_{1} \vee \gamma_{2}\right)$ by join of $\mathcal{A}_{1}$ and $\mathcal{A}_{2}$.

The join for baric algebras with idempotent of weight 1 was defined for Roberto Costa and H. Guzzo J. in [1]. Here, we extend this definition for dibaric algebras.

There exists a natural identification of $\mathrm{N}_{1}$, the dibarideal of $\mathcal{A}_{1}$ with the ideal of $\mathcal{A}_{1} \vee \mathcal{A}_{2}$ given by the set $\left\{(x, 0) \mid x \in \mathrm{N}_{1}\right\}$. Analogously, we identify $\mathrm{N}_{2}$, the dibarideal of $\mathcal{A}_{2}$, with the ideal of $\mathcal{A}_{1} \vee \mathcal{A}_{2}$ given by the set $\left\{(0, x) \mid x \in \mathrm{N}_{2}\right\}$. Take $u_{1}, v_{1} \in \mathcal{A}_{1}$ such that $\gamma_{1}\left(u_{1}\right)=\mathrm{m}$, $\gamma_{1}\left(v_{1}\right)=\mathrm{f}$, and $u_{2}, v_{2} \in \mathcal{A}_{2}$ such that $\gamma_{2}\left(u_{2}\right)=\mathrm{m}, \gamma_{2}\left(v_{2}\right)=\mathrm{f}$. We have that $u=\left(u_{1}, u_{2}\right)$ and $v=\left(v_{1}, v_{2}\right)$ are in $\mathcal{A}_{1} \vee \mathcal{A}_{2}$ with $\gamma_{1} \vee \gamma_{2}(u)=\mathrm{m}$ and $\gamma_{1} \vee \gamma_{2}(v)=\mathrm{f}$. Therefore, we write

$$
\mathcal{A}_{1} \vee \mathcal{A}_{2}=F u \oplus F v \oplus \mathrm{N}_{1} \oplus \mathrm{N}_{2}
$$

where $\mathrm{N}_{1} \oplus \mathrm{N}_{2}$ is the dibarideal of $\mathcal{A}_{1} \vee \mathcal{A}_{2}$.

Lemma 4.1. : The join of dibaric algebras satisfies the following properties :

(a) $\left(\S \vee \mathcal{A}, I d_{\mid \S} \vee \gamma\right) \cong(\mathcal{A}, \gamma)$; where $I d_{\mid \S}$ is the identity in $\S$;

(b) $(\S \vee \mathcal{A}, * \vee \gamma) \cong(\mathcal{A}, \gamma)$;

(c) $\left(\mathcal{A}_{1} \vee \mathcal{A}_{2}, \gamma_{1} \vee \gamma_{2}\right) \cong\left(\mathcal{A}_{2} \vee \mathcal{A}_{1}, \gamma_{2} \vee \gamma_{1}\right)$;

(d) $\left(\left(\mathcal{A}_{1} \vee \mathcal{A}_{2}\right) \vee \mathcal{A}_{3},\left(\gamma_{1} \vee \gamma_{2}\right) \vee \gamma_{3}\right) \cong\left(\mathcal{A}_{1} \vee\left(\mathcal{A}_{2} \vee \mathcal{A}_{3}\right), \gamma_{1} \vee\left(\gamma_{2} \vee \gamma_{3}\right)\right)$ 
In view of (d) we can define the join $\left(\bigvee_{i \in I} \mathcal{A}_{i}, \bigvee_{i \in I} \gamma_{i}\right)$ of an arbitrary family $\left\{\left(\mathcal{A}_{i}, \gamma_{i}\right)\right\}_{i \in I}$ of dibaric algebras, where $\bigvee_{i \in I} \mathcal{A}_{i}$ is the subalgebra of $\times_{i \in I} \mathcal{A}_{i}$, given by

$$
\bigvee_{i \in I} \mathcal{A}_{i}:=\left(\left(x_{i}\right)_{i \in I} \mid x_{i} \in \mathcal{A}_{i}, \gamma_{i}\left(x_{i}\right)=\gamma_{j}\left(x_{j}\right), \forall i, j \in I\right),
$$

and the dibaric weight homomorphism is given by $\left(\bigvee_{i \in I} \gamma_{i}\right)\left(\left(x_{i}\right)_{i \in I}\right):=$ $\gamma_{i}\left(x_{i}\right)$, where $i$ is a fixed and arbitrary index of $I$. Notice that for a family $\mathcal{A}_{1}, \ldots, \mathcal{A}_{r}$ of $r$ dibaric algebras with dimension of $\mathcal{A}_{i}$ equal to $n_{i}$, we have that

$$
\operatorname{dim} \bigvee_{i=1}^{r} \mathcal{A}_{i}=2(1-r)+\sum_{i=1}^{r} n_{i}
$$

A dibaric algebra $(\mathcal{A}, \gamma)$ is decomposable if there exist non-trivial dibaric algebras $\left(\mathcal{A}_{1}, \gamma_{1}\right)$ and $\left(\mathcal{A}_{2}, \gamma_{2}\right)$ such that $(\mathcal{A}, \gamma) \cong\left(\mathcal{A}_{1}, \gamma_{1}\right) \vee$ $\left(\mathcal{A}_{2}, \gamma_{2}\right)$. In another case, we say that $\mathcal{A}$ is indecomposable.

Lemma 4.2. A dibaric algebra $(A, \gamma)$ is decomposable if and only if $N$ is decomposable as $M(\mathcal{A})$ module.

Proof. Let $(\mathcal{A}, \gamma)$ be a decomposable dibaric algebra. Then, there exist two non-trivial dibaric algebras $\left(\mathcal{A}_{1}, \gamma_{1}\right)$ and $\left(\mathcal{A}_{2}, \gamma_{2}\right)$ and a dibaric isomorphism $f:(\mathcal{A}, \gamma) \longrightarrow\left(\mathcal{A}_{1} \vee \mathcal{A}_{2}, \gamma_{1} \vee \gamma_{2}\right)$. Since the dibarideal of $\mathcal{A}_{1} \vee \mathcal{A}_{2}$ is written as a direct sum of the ideals $\mathrm{N}_{1} \equiv\left\{(x, 0), x \in \mathrm{N}_{1}\right\}$ and $\mathrm{N}_{2} \equiv\left\{(0, x), x \in \mathrm{N}_{2}\right\}$ and $f$ is a dibaric isomorphism, it follows that the dibarideal $\mathrm{N}$ of $\mathcal{A}$ is written as the direct sum of the non-trivial ideals $f^{-1}\left(\mathrm{~N}_{1}\right)$ and $f^{-1}\left(\mathrm{~N}_{2}\right)$. Therefore $\mathrm{N}$ is decomposable.

Conversely, let $(\mathcal{A}, \gamma)$ be a dibaric algebra such that $\mathcal{N}$ is decomposable as $\mathrm{M}$ modulo. Then, there exist $\mathrm{N}_{1}, \mathrm{~N}_{2}$, two proper $\mathrm{M}$ submodules of $\mathrm{N}$ such that $\mathrm{N}=\mathrm{N}_{1} \oplus \mathrm{N}_{2}$. So, we can write $\mathcal{A}=$ $F u \oplus F v \oplus \mathrm{N}_{1} \oplus \mathrm{N}_{2}$ where $u, v$ satisfy $\gamma(u)=\mathrm{m}$ and $\gamma(v)=\mathrm{h}$. Notice that the subspaces $\mathrm{N}_{1}$ and $\mathrm{N}_{2}$ are ideals of $\mathcal{A}$. Then, an element $x \in \mathcal{A}$ is uniquely written as a sum $x=\alpha u+\beta v+x_{1}+x_{2}$, where $\alpha, \beta \in F$ and $x_{1} \in \mathrm{N}_{1}, x_{2} \in \mathrm{N}_{2}$. This decomposition give us the means to define 
the projections $\pi_{i}: \mathcal{A} \longrightarrow \mathrm{N}_{i}, \pi_{i}(x)=x_{i}$, for $i=1,2$. The mappings $\pi_{i},(i=1,2)$ satisfy the following properties:

$\pi_{i}(u x)=u \pi_{i}(x), \quad \pi_{i}(x u)=\pi_{i}(x) u, \quad \pi_{i}(v x)=v \pi_{i}(x), \quad \pi_{i}(x v)=\pi_{i}(x) v$, and $\pi_{i}(x y)=\pi_{i}(x) \pi_{i}(y)$ for all $x, y \in \mathrm{N}$. Now, we define over the vector space $\mathcal{A}_{i}=F u_{i} \oplus F v_{i} \oplus \mathrm{N}_{i},(i=1,2)$ a product " ." such that, restricted to $\mathrm{N}_{i}$, it coincides with the multiplication of $\mathrm{N}_{i}$ as a subalgebra of $\mathcal{A}$ that is $x_{i} \cdot y_{i}=x_{i} y_{i}$ for $x_{i}, y_{i} \in \mathrm{N}_{i}$ and

$$
\begin{array}{ll}
u_{i} \cdot v_{i}=\frac{\left(u_{i}+v_{i}\right)}{2}+\pi_{i}(u v), & v_{i} \cdot u_{i}=\frac{\left(u_{i}+v_{i}\right)}{2}+\pi_{i}(v u), \\
u_{i} \cdot u_{i}=\pi_{i}\left(u^{2}\right), & v_{i} \cdot v_{i}=\pi_{i}\left(v^{2}\right), \\
x_{i} \cdot u_{i}=x_{i} u, & u_{i} \cdot x_{i}=u x_{i}, \\
x_{i} \cdot v_{i}=x_{i} v, & v_{i} \cdot x_{i}=v x_{i} .
\end{array}
$$

where $x_{i}, y_{i} \in \mathrm{N}_{i}$. The algebra $\left(\mathcal{A}_{i}, \cdot\right)$ has dibaric homomorphism given by $\gamma_{i}\left(\alpha u_{i}+\beta v_{i}+x_{i}\right)=\alpha \mathrm{m}+\beta \mathrm{f}$, for all $\alpha, \beta \in F$ and $x_{i} \in \mathrm{N}_{i}$. So, $\left(\mathcal{A}_{1}, \gamma_{1}\right)$ and $\left(\mathcal{A}_{2}, \gamma_{2}\right)$ are dibaric algebras and its join $\left(\mathcal{A}_{1}, \gamma_{1}\right) \vee\left(\mathcal{A}_{2}, \gamma_{2}\right)$ is isomorphic to $(\mathcal{A}, \gamma)$. To see that this last assertion is true, we consider the mapping $f: \mathcal{A} \longrightarrow \mathcal{A}_{1} \vee \mathcal{A}_{2}$, given by

$$
f(\alpha u+\beta v+x)=\left(\alpha u_{1}+\beta v_{1}+\pi_{1}(x), \alpha u_{2}+\beta v_{2}+\pi_{2}(x)\right) .
$$

Simple computations show that $f$ is a dibaric isomorphism.

The above result can be generalized in the following sense: if a dibaric algebra $(\mathcal{A}, \gamma)$ is written as join of a family of dibaric algebras $\left\{\left(\mathcal{A}_{i}, \gamma_{i}\right)\right\}_{i=i}^{n}$, that is $(\mathcal{A}, \gamma)=\left(\bigvee_{i=1}^{n} \mathcal{A}_{i}, \bigvee_{i=1}^{n} \gamma_{i}\right)$, then we identify $\mathrm{N}_{j} \equiv$ $\left\{\left(0, \ldots, x_{j}, \ldots, 0\right) \in \bigvee_{i=1}^{n} \mathcal{A}_{i}, x_{j} \in \mathrm{N}_{j}\right\}$, where $\mathrm{N}_{j}=\operatorname{ker}\left(\gamma_{j}\right)$ and we have that the dibaric ideal $\mathrm{N}$ of $\mathcal{A}$ is written as a direct sum of the $\mathrm{M}$ submodules as follows $\mathrm{N}=\mathrm{N}_{1} \oplus \cdots \oplus \mathrm{N}_{n}$.

Conversely, if we have that the dibaric ideal $\mathrm{N}$ of an algebra $(\mathcal{A}, \gamma)$ is written as a direct sum of $\mathrm{M}$ submodules of $\mathrm{N}$, that is $\mathrm{N}=\bigoplus_{i=1 \mathrm{~N} i}^{n}$, where $\mathrm{N}_{i}$ is $\mathrm{M}$ submodule, then for each index $i$ we define an algebra over the vector space $\mathcal{A}_{i}=F u_{i} \oplus F v_{i} \oplus \mathrm{N}_{i}$ with a product as in the above lemma where $\pi_{i}\left(\alpha u_{i}+\beta v_{i}+x\right)=x_{i}$ whenever $x=\sum_{j=1}^{n} x_{j}$ with $x_{j} \in \mathrm{N}_{j}$. This algebra has weight homomorphisms given by $\gamma_{i}\left(\alpha u_{i}+\beta v_{i}+\pi_{i}(x)\right)=\alpha \mathrm{m}+\beta \mathrm{f}$. So, $\left(\mathcal{A}_{i}, \gamma_{i}\right)$ is a dibaric algebras and $f:(\mathcal{A}, \gamma) l\left(\bigvee_{i=1}^{n} \mathcal{A}_{i}, \bigvee_{i=1}^{n} \gamma_{i}\right)$, defined by

$$
f(\alpha u+\beta v+x)=\left(\alpha u_{1}+\beta v_{1}+\pi_{1}(x), \ldots, \alpha u_{n}+\beta v_{n}+\pi_{n}(x)\right),
$$


where $\alpha, \beta \in F, x \in \mathrm{N}$, is a dibaric homomorphism.

Corollary 4.1. : If a dibaric algebra $(\mathcal{A}, \gamma)$ is written as a join of a finite family of dibaric algebras, that is, $(\mathcal{A}, \gamma) \cong\left(\bigvee_{i=1}^{n} A_{i}, \bigvee_{i=1}^{n} \gamma_{i}\right)$, then $N \cong N_{1} \times \cdots \times N_{n}$, where $N_{i}:=\operatorname{ker}\left(\gamma_{i}\right)$. Conversely, if the dibaric ideal $N$ of an arbitrary dibaric algebra $(\mathcal{A}, \gamma)$, is written as a direct sum of ideals $I_{1}, \ldots, I_{n}$, then there exist dibaric algebras $\left\{\left(\mathcal{A}_{i}, \gamma_{i}\right)\right\}_{i=1}^{n}$, with $\operatorname{ker}\left(\gamma_{i}\right) \cong I_{i}$, such that $(\mathcal{A}, \gamma) \cong\left(\bigvee_{i=1}^{n} \mathcal{A}_{i}, \bigvee_{i=1}^{n} \gamma_{i}\right)$.

We say that a dibaric algebra $(\mathcal{A}, \gamma)$ satisfies the ascendent chain condition (a.c.c.) if $\mathrm{N}=\operatorname{ker}(\gamma)$ satisfies (a.c.c.) as $\mathrm{M}$ module, where $\mathrm{M}$ is the multiplication algebra of $\mathcal{A}$. Analogously, we say that a dibaric algebra $(\mathcal{A}, \gamma)$ satisfy the descendent chain condition (d.c.c.) if $\mathrm{N}$ satisfy (d.c.c.) as $\mathrm{M}(\mathcal{A})$ module.

Lemma 4.3. : Let $\left(\mathcal{A}_{1} \vee \mathcal{A}_{2}, \gamma_{1} \vee \gamma_{2}\right)$ be the join of two dibaric algebras $\left(\mathcal{A}_{1}, \gamma_{1}\right)$ and $\left(\mathcal{A}_{2}, \gamma_{2}\right)$. Then $\left(e_{1}, e_{2}\right)$ is a semiprincipal idempotent in $\mathcal{A}_{1} \vee \mathcal{A}_{2}$ if and only if $e_{1}, e_{2}$ are semiprincipal idempotent elements in $\mathcal{A}_{1}$ and $\mathcal{A}_{2}$, respectively. Therefore, it follows that $\mathcal{A}_{1} \vee \mathcal{A}_{2}$ has a semiprincipal idempotent if and only if $\mathcal{A}_{1}$ and $\mathcal{A}_{2}$ have a semiprincipal idempotent.

Proof. $(\Rightarrow)$ Let $e:=\left(e_{1}, e_{2}\right)=\left(u_{1}, u_{2}\right)+\left(v_{1}, v_{2}\right)$ be a semiprincipal idempotent element in $\mathcal{A}_{1} \vee \mathcal{A}_{2}$, with $u_{1}, v_{1} \in \mathcal{A}_{1}$ and $u_{2}, v_{2} \in \mathcal{A}_{2}$. Under these conditions, we have that $\left(u_{1}, u_{2}\right)^{2}=(0,0)$. So, it follows that $\left(u_{1}^{2}, u_{2}^{2}\right)=(0,0)$, therefore $u_{1}^{2}=0$ and $u_{2}^{2}=0$. Analogously, $\left(v_{1}, v_{2}\right)^{2}=(0,0)$, and so $v_{1}^{2}=0$ and $v_{2}^{2}=0$. On the other hand, $\left(u_{1}, u_{2}\right)\left(v_{1}, v_{2}\right)=\left(u_{1} v_{1}, u_{2} v_{2}\right)=\left(\left(u_{1}, u_{2}\right)+\left(v_{1}, v_{2}\right)\right) / 2$. Then, $u_{1} v_{1}=$ $\left(u_{1}+v_{1}\right) / 2$ and $u_{2} v_{2}=\left(u_{2}+v_{2}\right) / 2$. Finally, we have $\left(\gamma_{1} \vee \gamma_{2}\right)\left(u_{1}, u_{2}\right)=$ $\gamma_{1}\left(u_{1}\right)=\gamma_{2}\left(u_{2}\right)=\mathrm{m}$ and $\left(\gamma_{1} \vee \gamma_{2}\right)\left(v_{1}, v_{2}\right)=\gamma_{1}\left(v_{1}\right)=\gamma_{2}\left(v_{2}\right)=\mathrm{f}$. Hence, $e_{1}=u_{1}+v_{1}$ and $e_{2}=u_{2}+v_{2}$ are semiprincipal idempotent elements in $\mathcal{A}_{1}$ and $\mathcal{A}_{2}$, respectively.

$(\Leftarrow)$ If $e_{1}=u_{1}+v_{1} \in \mathcal{A}_{1}$ and $e_{2}=u_{2}+v_{2} \in \mathcal{A}_{2}$ are semiprincipal idempotent elements, the ordered pair $\left(e_{1}, e_{2}\right)=\left(u_{1}, u_{2}\right)+\left(v_{1}, v_{2}\right) \in$ $\mathcal{A}_{1} \vee \mathcal{A}_{2}$ because $\gamma_{1}\left(e_{1}\right)=\mathrm{m}+\mathrm{f}=\gamma_{2}\left(e_{2}\right)$. This element satisfy 
$\left(e_{1}, e_{2}\right)^{2}=\left(e_{1}^{2}, e_{2}^{2}\right)=\left(e_{1}, e_{2}\right)$ and $\left(u_{1}, u_{2}\right)^{2}=\left(u_{1}^{2}, u_{2}^{2}\right)=(0,0),\left(v_{1}, v_{2}\right)^{2}=$ $\left(v_{1}^{2}, v_{2}^{2}\right)=(0,0)$. Finally, we have that $\left(u_{1}, u_{2}\right)\left(v_{1}, v_{2}\right)=\left(u_{1} v_{1}, u_{2} v_{2}\right)=$ $\left(u_{1}, u_{2}\right) / 2+\left(v_{1}, v_{2}\right) / 2$. Therefore $\left(e_{1}, e_{2}\right)$ is a semiprincipal idempotent in $\mathcal{A}_{1} \vee \mathcal{A}_{2}$.

The above result can be generalized in the following sense: if $(\mathcal{A}, \gamma)$ has a semiprincipal idempotent $e=u+v$ and this algebra is isomorphic to the join of a finite family $\left\{\left(\mathcal{A}_{i}, \gamma_{i}\right)\right\}_{i=1}^{n}$ of dibaric subalgebras, then each algebra $\mathcal{A}_{i}$ has a semiprincipal idempotent $e_{i}=u_{i}+v_{i}$. To prove it, we use induction over $n$ and the associativity of the join of dibaric algebras. Therefore, we have the corollary below.

Corollary 4.2. : If $(A, \gamma) \cong\left(\bigvee_{i=1}^{n} \mathcal{A}_{i}, \bigvee_{i=1}^{n} \gamma_{i}\right)$, then $\mathcal{A}$ has a semiprincipal idempotent if and only if $\mathcal{A}_{i}$ have semiprincipal idempotents, for all $i$.

Lemma 4.4. : Let $(\mathcal{A}, \gamma)$ be a dibaric algebra with $e=u+v$ as semiprincipal idempotent element such that $(\mathcal{A}, \gamma) \cong\left(\bigvee_{i=1}^{n} \mathcal{A}_{i}, \bigvee_{i=1}^{n} \gamma_{i}\right)$. Then for every $i$, there exists a dibaric subalgebra of $\mathcal{A}$ isomorphic to $\left(A_{i}, \gamma_{i}\right)$ with $e=u+v$ as semiprincipal idempotent.

Proof. We will suppose that $(\mathcal{A}, \gamma)=\left(\bigvee_{i=1}^{n} \mathcal{A}_{i}, \bigvee_{i=1}^{n} \gamma_{i}\right)$. If $e=$ $u+v$ is a semiprincipal idempotent element in $\bigvee_{i=1}^{n} \mathcal{A}_{i}$, with

$$
e=\left(e_{1}, \ldots, e_{n}\right), \quad u=\left(u_{1}, \ldots, u_{n}\right)=u \quad \text { and } \quad v=\left(v_{1}, \ldots, v_{n}\right),
$$

then $e_{i}=u_{i}+v_{i}$ is a semiprincipal idempotent in $\mathcal{A}_{i}$. On the other hand $\bigvee_{i=1}^{n} \mathcal{A}_{i}$ can be written as

$$
\bigvee_{i=1}^{n} \mathcal{A}_{i}=F u \oplus F v \oplus \mathrm{N}_{1} \oplus \cdots \oplus \mathrm{N}_{n}
$$

where

$$
\mathrm{N}_{j}=\left\{\left(0, \ldots, x_{j}, \ldots, 0\right) \in \bigvee_{i=1}^{n} \mathcal{A}_{i} \mid x_{j} \in \operatorname{ker}\left(\gamma_{j}\right)\right\}
$$

is an barideal of $\bigvee_{i=1}^{n} \mathcal{A}_{i}$. So, since $e=u+v$ is a semiprincipal idempotent element and $\mathrm{N}_{j}$ is a barideal of $\mathcal{A}$, for $j=1,2, \ldots, n$, it 
follows that $F u \oplus F v \oplus \mathrm{N}_{j}$ is a dibaric subalgebra of $\bigvee_{i=1}^{n} \mathcal{A}_{i}$. Finally, the linear mapping $f_{j}: F u \oplus F v \oplus \mathrm{N}_{j} \longrightarrow \mathcal{A}_{j}$ defined by $f_{j}(\alpha u+\beta v+x)=\alpha u_{j}+\beta v_{j}+x$ for all $\alpha, \beta \in F$ e $x \in \mathrm{N}_{j}$ is a dibaric isomorphism.

Lemma 4.5. : If a dibaric algebra $(\mathcal{A}, \gamma)$ with semiprincipal idempotent $e=u+v$ satisfying the descendent chain condition, then there exists a finite number of indecomposable dibaric subalgebras $\left\{\left(\mathcal{A}_{i}, \gamma_{i}\right)\right\}_{i=1}^{n}$ of $(\mathcal{A}, \gamma)$, such that $(\mathcal{A}, \gamma) \cong\left(\mathcal{A}_{1} \vee \cdots \vee \mathcal{A}_{n}, \gamma_{1} \vee \cdots \vee \gamma_{n}\right)$.

Proof. Since $(\mathcal{A}, \gamma)$ satisfy the descendent chain condition, it follows that $\mathrm{N}$ satisfies d.c.c. as $\mathrm{M}(\mathcal{A})$ module. So, there exist indecomposable $\mathrm{M}(\mathcal{A})$ submodules $\mathrm{N}_{1}, \ldots, \mathrm{N}$ of $\mathrm{N}$ such that $\mathrm{N}=$ $\mathrm{N}_{1} \oplus \mathrm{N}_{2} \oplus \cdots \oplus \mathrm{N}_{m}$. Therefore, for each $j, \mathcal{A}_{j}=F u \oplus F v \oplus \mathrm{N}_{j}$ is a dibaric subalgebra of $\mathcal{A}$ such that $(\mathcal{A}, \gamma) \cong\left(\bigvee_{i=1}^{n} \mathcal{A}_{i}, \bigvee_{i=1}^{n} \gamma_{i}\right)$. Finally, we will show that $\mathcal{A}_{j}$ is indecomposable, for each $j$. We observe that a dibaric ideal $I$ of $\mathcal{A}_{j}$ is a dibaric ideal of $\mathcal{A}$, because $I \mathcal{A}=I\left(F u \oplus F v \oplus \mathrm{N}_{1} \oplus \cdots \oplus \mathrm{N}_{n}\right)=I \mathcal{A}_{j} \subseteq I$. Analogously $\mathcal{A} I \subseteq I$. So, the $\mathrm{M}(\mathcal{A})$ submodules of $\mathrm{N}_{j}$ are equal to the $\mathrm{M}(\mathcal{A})$ submodules of $\mathrm{N}_{j}$. Since $\mathrm{N}_{j}$ is indecomposable as $\mathrm{M}(\mathcal{A})$-module, it follows that $\mathrm{N}_{j}$ is indecomposable as $\mathrm{M}\left(\mathcal{A}_{j}\right)$-module. So, $\mathcal{A}_{j}$ is indecomposable.

Theorem 4.1. : (Krull-Schmidt) Let $(\mathcal{A}, \gamma)$ be a dibaric algebra with semiprincipal idempotent element $e=u+v$ that satisfies d.c.c. and a.c.c.. If

$$
(\mathcal{A}, \gamma)=\left(\mathcal{A}_{1} \vee \cdots \vee \mathcal{A}_{n}, \gamma_{1} \vee \cdots \vee \gamma_{n}\right),(\mathcal{A}, \gamma)=\left(B_{1} \vee \cdots \vee B_{m}, \chi_{1} \vee \cdots \vee \chi_{m}\right)
$$

where each $\left(\mathcal{A}_{i}, \gamma_{i}\right)$ and $\left(B_{j}, \chi_{j}\right)$ are indecomposable dibaric subalgebras of $(\mathcal{A}, \gamma)$, then $n=m$ and reindexing, we have that $\left(\mathcal{A}_{i}, \gamma_{i}\right) \cong$ $\left(B_{i}, \chi_{i}\right)$, for each $i \in\{1, \ldots, n\}$.

Proof. Since we have two decompositions of $\mathcal{A}$ in indecomposable dibaric subalgebras, then $\mathrm{N}=\operatorname{ker}(\gamma)$ is decomposed in indecomposable M submodules as follows

$$
\mathrm{N}=\mathrm{N}_{1} \oplus \cdots \oplus \mathrm{N}_{n}, \quad \mathrm{~N}=\mathcal{P}_{1} \oplus \cdots \oplus \mathcal{P}_{m}
$$


where $\mathrm{N}_{i}=\operatorname{ker}\left(\gamma_{i}\right)$ and $\mathcal{P}_{j}=\operatorname{ker}\left(\chi_{j}\right)$. According to the KrullSchmidt's Theorem for $\mathcal{A}$ modules, we have that $m=n$ and with a reindexation $\mathrm{N}_{i} \cong \mathcal{P}_{i}$ as $\mathcal{A}$-modules. By the same Theorem, we can write

$$
\mathrm{N}=\mathrm{N}_{1} \oplus \mathrm{N}_{2} \oplus \cdots \oplus \mathrm{N}_{k} \oplus \mathcal{P}_{k+1} \oplus \cdots \oplus \mathcal{P}_{n}
$$

for $0 \leq k \leq n$. Let $j$ be a fixed index. First we will show that $\mathrm{N}_{j} \cong \mathcal{P}_{j}$ as algebras. We consider the two decomposition of (4.1)

$$
\begin{aligned}
& \mathrm{N}_{1} \oplus \mathrm{N}_{2} \oplus \cdots \oplus \mathrm{N}_{j-1} \oplus \mathrm{N}_{j} \oplus \mathcal{P}_{j+1} \oplus \cdots \oplus \mathcal{P}_{n} \\
& \mathrm{~N}_{1} \oplus \mathrm{N}_{2} \oplus \cdots \oplus \mathrm{N}_{j-1} \oplus \mathcal{P}_{j} \oplus \mathcal{P}_{j+1} \oplus \cdots \oplus \mathcal{P}_{n} .
\end{aligned}
$$

So, if $x \in \mathrm{N}$, then $x$ can be written in two different ways

$$
x=x_{1}+\cdots+x_{n}, \quad x=x_{1}^{\prime}+\cdots+x_{n}^{\prime}
$$

where $x_{j} \in \mathrm{N}_{j} ; x_{j}^{\prime} \in \mathcal{P}_{j} ; x_{r}, x_{r}^{\prime} \in \mathrm{N}_{r}$ for $1 \leq r \leq j-1$, and $x_{s}, x_{s}^{\prime} \in \mathcal{P}_{s}$ for $j+1 \leq s \leq n$. Let $\tau_{j}$ be the injection of $\mathrm{N}_{j}$ in $\mathrm{N}$ and $\pi_{j}: \mathrm{N} \longrightarrow$ $\mathcal{P}_{j}$ the projection define via $\pi_{j}(x):=x_{j}^{\prime}$ for all $x \in \mathrm{N}$. Then the composition $p_{j}:=\pi_{j} \circ \tau_{j}$ of $\mathrm{N}_{j}$ in $\mathcal{P}_{j}$ is an isomorphism of algebras. For $x, y \in \mathrm{N}_{j}$ we have that $p_{j}(x y)=(x y)_{j}^{\prime}=\left\{\left(x_{1}^{\prime}+\cdots+x_{n}^{\prime}\right)\left(y_{1}^{\prime}+\right.\right.$ $\left.\left.\cdots+y_{n}^{\prime}\right)\right\}_{j}^{\prime}=\left\{x_{1}^{\prime} y_{1}^{\prime}+\cdots+x_{n}^{\prime} y_{n}^{\prime}\right\}_{j}^{\prime}=x_{j}^{\prime} y_{j}^{\prime}=p_{j}(x) p_{j}(y)$ and hence $p_{j}$ is a homomorphism of algebras. By to prove that $p_{j}$ is injective, we consider $x \in \operatorname{ker}\left(p_{j}\right)$. Then $0=p_{j}(x)=x_{j}^{\prime}$ and so, $x \in \mathrm{N} \cap\left(\mathrm{N}_{1} \oplus \mathrm{N}_{2} \oplus\right.$ $\left.\cdots \oplus \mathrm{N}_{j-1} \oplus \mathcal{P}_{j+1} \oplus \cdots \oplus \mathcal{P}_{n}\right)=\{0\}$. Therefore $x=0$. Next, by to prove that $p_{j}$ is onto, we take $y \in \mathcal{P}_{j}$. Then, $y=y_{j}^{\prime}=\pi_{j}(y)=\pi_{j}\left(y_{1}+\right.$ $\left.\cdots+y_{n}\right)=\pi_{j}\left(y_{1}\right)+\cdots+\pi_{j}\left(y_{n}\right)=0+\cdots 0+\pi_{j}\left(y_{j}\right)+0+\cdots+0=\pi_{j}\left(y_{j}\right)$ where, according to above decomposition, $y_{j} \in \mathrm{N}_{j}$.

Finally, we will define a dibaric isomorphism between the algebras $\mathcal{A}_{j}$ and $\mathrm{B}_{j}$. According to Lemma 4.4 we can assume, without lost of generality, that $\left(\mathcal{A}_{j}, \gamma_{j}\right)$ and $\left(\mathrm{B}_{j}, \chi_{j}\right)$ have the same semiprincipal idempotent element denoted by $e=u+v$. Then, we define the application $f_{j}: \mathcal{A}_{j} \longrightarrow \mathrm{B}_{j}$, by

$$
f_{j}(\alpha u+\beta v+x)=\alpha u+\beta v+p_{j}(x)
$$

where $\alpha, \beta \in F$ e $x \in \mathrm{N}_{j}$. It is clear that $f_{j}$ is a linear isomorphism and also that $\gamma_{j}=\chi_{j} \circ f_{j}$. Therefore, only rest to show that $f_{j}$ is 
a homomorphism of algebras. Notice that if $w \in\langle u, v\rangle$ and $x \in \mathrm{N}_{j}$, then

$$
p_{j}(w x)=w p_{j}(x), \quad p_{j}(x w)=p_{j}(x) w,
$$

because $p_{j}(w x)=\left\{w\left(x_{1}^{\prime}+\cdots+x_{n}^{\prime}\right)\right\}_{j}^{\prime}=\left\{w x_{1}^{\prime}+\cdots+w x_{n}^{\prime}\right\}_{j}^{\prime}=w x_{j}^{\prime}=$ $w p_{j}(x)$. Analogously, we have the other equality. So, if $a=\alpha u+\beta v+x$, $b=\eta u+\mu v+y$ are in $\mathcal{A}_{j}$, then

$$
\begin{array}{r}
f_{j}(a b)=f_{j}\left(\frac{1}{2}(\alpha \mu+\beta \eta)(u+v)+\alpha u y+\beta v y+\eta x u+\mu x v+x y\right) \\
=\frac{1}{2}(\alpha \mu+\beta \eta)(u+v)+\alpha p_{j}(u y)+\beta p_{j}(v y)+\eta p_{j}(x u)+ \\
\mu p_{j}(x v)+p_{j}(x y) \\
=\frac{1}{2}(\alpha \mu+\beta \eta)(u+v)+\alpha u p_{j}(y)+\beta v p_{j}(y)+\eta p_{j}(x) u+ \\
\mu p_{j}(x) v+p_{j}(x) p_{j}(y) \\
=\left(\left(\alpha u+\beta v+p_{j}(x)\right)\left(\eta u+\mu v+p_{j}(y)\right)=f_{j}(a) f_{j}(b) .\right.
\end{array}
$$

\section{References}

[1] R. Costa and H. Jr Guzzo, Indecomposable baric algebras, Linear Algebra Appl. 183, pp. 223-236, (1993).

[2] R. Costa and H. Jr Guzzo, Indecomposable baric algebras II, Linear Algebra Appl. 196, pp. 233-242, (1994).

[3] I.M.H. Etherington, On non-associative combinations, Proc. Edinb. Math. Soc. 59, pp. 153-162, (1939).

[4] P. Holgate, Genetic Algebras associated with sex linkage, Proc. Edinb. Math. Soc. 17, pp. 113-120, (1970).

[5] Y. Lyubich, Mathematical Structure in Populations Genetics, Springer-Verlag, (1983).

[6] M. Lynn Reed, Algebraic structures of genetic inheritance, Bull. Amer. Math. Soc (N. S.) 34, No. 2, pp. 107-131, (1997).

[7] A. Wörz-Busekros, Algebras in Genetics, Springer-Verlag vol. 36, (1980). 
[8] A. Wörz-Busekros, The zygotic algebra for sex linkage, J. Math. Biol. 1, pp. 37-46, (1974).

[9] A. Wörz-Busekros, The zygotic algebra for sex linkage II, J. Math. Biol. 2, pp. 359-371, (1975).

Received : April 2000.

\section{María Aparecida Couto}

Departamento de Matemática

Universidade Federal do Rio Grande do Norte

Caixa Postal 1524 - Campus Universitário

Lagoa Nova - CEP 59072-970

Natal - RN

Brasil

\section{Juan Carlos Gutiérrez Fernández}

Departamento de Matemática

Instituto de Matemática e Estatística

Universidade de São Paulo

Caixa Postal 66.281

São Paulo - SP, 05315-970

Brasil

e-mail : jcgf@ime.usp.br 